\title{
Sequential postoperative intraperitoneal chemotherapy for colorectal cancer with peritoneal metastases: a narrative review
}

\author{
Peter H. Cashin, Wilhelm Graf \\ Department of Surgical Sciences, Section of Surgery, Uppsala University, Akademiska Sjukhuset, Uppsala, Sweden \\ Contributions: (I) Conception and design: All authors; (II) Administrative support: All authors; (III) Provision of study materials or patients: All \\ authors; (IV) Collection and assembly of data: All authors; (V) Data analysis and interpretation: All authors; (VI) Manuscript writing: All authors; (VII) \\ Final approval of manuscript: All authors. \\ Correspondence to: Dr. Peter Cashin. Department of Surgical Sciences, Bengt Ihre Fellow, Uppsala University, Akademiska Sjukhuset, 75185 Uppsala, \\ Sweden. Email: Peter.cashin@surgsci.uu.se.
}

\begin{abstract}
Sequential postoperative intraperitoneal chemotherapy (SPIC) is a chemotherapy abdominal infusion given as a postoperative adjuvant treatment for 6 months after cytoreductive surgery (CRS) for peritoneal surface malignancies. It has most commonly been used in conjunction with ovarian cancer where the SPIC treatment has been integrated with adjuvant systemic chemotherapy. This review investigates the role of SPIC in the setting of colorectal cancer with peritoneal metastases. The focus is on the CRS+SPIC combination treatment with no systemic chemotherapy component. Several cohort studies, several comparative studies, and one randomized trial have been reported with several important endpoints. The following aspects will be covered in this review: overall survival, disease-free survival, morbidity, quality-oflife, and cost-effectiveness. In comparison to systemic chemotherapy alone for isolated resectable colorectal peritoneal metastases, CRS+SPIC is superior concerning overall survival, has no difference in morbidity, is similar in quality-of-life, and SPIC is cost-effective. In comparison to HIPEC, results are conflicting in multivariate analysis; but in a univariate analysis HIPEC (most often combined with systemic adjuvant therapy) appears superior to SPIC alone (no systemic component). The future of SPIC is uncertain. However, a combination of HIPEC and SPIC \pm a systemic chemotherapy component is a possible direction to explore further.
\end{abstract}

Keywords: Colorectal cancer; peritoneal metastases; cytoreductive surgery (CRS); intraperitoneal chemotherapy; 5-fluorouracil; HIPEC; early intraperitoneal chemotherapy (EPIC)

Submitted Mar 02, 2020. Accepted for publication Aug 17, 2020.

doi: 10.21037/jgo-20-137

View this article at: http://dx.doi.org/10.21037/jgo-20-137

\section{Introduction \& methodology}

Locoregional chemotherapy to treat peritoneal metastases has great pharmacodynamic advantages over systemic treatment but also definite limitations. A major challenge is the limited penetration into tissue-at most a few mm (1). Selection is required only of patients with minimal residual disease as suitable candidates for locoregional treatment. The most common treatment package today is cytoreductive surgery (CRS) combined with hyperthermic intraoperative intraperitoneal chemotherapy, i.e., HIPEC (2). In this well-established modality, maximum cytoreduction is combined with a single chemotherapy perfusion in an abdomen released from all adhesions allowing extensive exposure of the abdominal compartment to the heated chemotherapy solution. However, limited time of treatment may be a disadvantage leading to a higher risk of tumor regrowth. In order to overcome this problem, several other locoregional protocols have been developed. Adding a few days treatment after a major peritonectomy procedure is achieved when early intraperitoneal chemotherapy (EPIC) is used. Here normothermic treatment follows surgery 
for 4-6 days postoperatively. The drug is infused through an intraperitoneal catheter placed prior to closure of the abdomen (3). A new modality is pressurized intraperitoneal chemotherapy (PIPAC) which has been used for various gastrointestinal malignancies with peritoneal spread (4). PIPAC treatment is usually repeated, mimicking systemic oncologic treatment but as a locoregional treatment in the abdominal cavity. This methodology requires a laparoscopic procedure for its application, thus making it a costly treatment alternative.

Another way of repeating locoregional treatment in the abdomen is sequential intraperitoneal chemotherapy (SPIC) which has been used for colorectal cancer with peritoneal metastases as a 6-month adjuvant intraperitoneal treatment after CRS (5). In the present review article, we describe the development of the method of delivering SPIC, treatment administration, morbidity/mortality, oncological results, SPIC $v s$. HIPEC, and the future of SPIC.

This review was conducted using literature relevant to SPIC treatment development and clinical results for colorectal cancer from 1989 to 2018. We have used only published peer reviewed articles in the English language.

We present the following article in accordance with the Narrative Review Checklist (available at http://dx.doi. org/10.21037/jgo-20-137).

\section{Development of the spic method}

A one-week course of intraportal infusion as adjuvant therapy after curative resection of colorectal cancer was popularized during the 1980s. In order to avoid a portal vein catheter, intraperitoneal infusion was suggested (6). A randomized controlled study suggested that 5 -fluorouracil at a dose of $500 \mathrm{mg} / \mathrm{m}^{2} /$ day combined with intravenous leucovorine $60 \mathrm{mg} / \mathrm{m}^{2} /$ day for 6 consecutive postoperative days could be given without increased risk of postoperative complications after colon cancer surgery (7). In an animal model, resembling adjuvant use of 5-fluorouracil, anastomotic strength was reduced indicating that the dose used was close to the threshold of impaired anastomotic healing (8). Moreover, collagen accumulation in humans was reduced during EPIC treatment to a similar extent, but normalizes after the end of treatment (9). Based on these experiments, the SPIC protocol was developed as a repeated EPIC variant. In order to avoid catheter related problems an abdominal Port a Cath was used. The treatment was designed to resemble an adjuvant course of intravenous 5 -flurouracil. SPIC is administered as an intraperitoneal dose $\left(550 \mathrm{mg} / \mathrm{m}^{2} 5\right.$-fluorouracil) a day for 6 days in a row and then repeated every 4-6 weeks over a 6-month period usually 6 cycles. The most common currently used protocol is the 5 -fluorouracil (5-FU) protocol.

\section{Administration of chemotherapy solution and morbidity \& mortality}

CRS+SPIC treatment has been compared to systemic chemotherapy alone in one RCT (10). This was at a time when peritoneal metastases from colorectal cancer was still considered a generalized form of colorectal cancer and systemic chemotherapy was still the international standard as first line therapy. The ability to complete all planned cycles in both groups was impaired. In the CRS+SPIC group, $33 \%$ completed all 6 cycles of postoperative SPIC therapy with a mean of 3.6 cycles/person. In the systemic chemotherapy arm, $58 \%$ completed all 12 cycles with a mean of 10 cycles/person. Grade 3-4 adverse events was numerically higher in the systemic chemotherapy group at $50 \%$ vs. $42 \%$ in the SPIC group. There was no mortality in either group. In a non-comparison study on just CRS+SPIC, the grade 3-4 morbidity was $41 \%$ (51/123 patients) and there were 5 deaths within 90 days (4\%) (11).

The morbidity and mortality of CRS+SPIC is acceptable. The main problem has been the feasibility of completing all planned cycles. Due to adhesion development, there is a significant problem with port dysfunction (10-12). This is also something that is well known from the ovarian cancer setting (13). Problems with port dysfunction leads to premature treatment termination. In the colorectal RCT, there was a significant incentive to complete as many treatments as possible (10). It is quite likely that the number of successful SPIC cycles will be less in the routine clinical setting. However, there are no studies showing how many cycles are needed to reach a clinically relevant locoregional effect.

Another limitation with SPIC is the variable distribution of chemotherapy solution in the abdomen and pelvis as measured with single photon emission tomography. The fluid distribution correlated with the number of cycles that could be given (14). In the non-randomized comparison, the distribution volume varied from $32 \mathrm{~mL}$ to almost $12,000 \mathrm{~mL}$ with an average distribution of 2,896 mL (12). 
This leads to dosing/toxicity issues as the uptake may vary accordingly.

\section{Survival benefit}

The SPIC method has not been widely used as a longterm postoperative normothermic intraperitoneal adjuvant therapy. It has, however, been extensively researched within the area of ovarian cancer. In this setting, there are many randomized trials proving the survival benefit of this approach (13). For colorectal cancer peritoneal metastases treatment, there is only one randomized trial as mentioned above (10). This trial demonstrated that the combined CRS+SPIC approach was more beneficial than systemic chemotherapy alone. The median OS was 25 vs. 18 months, $\mathrm{P}=0.04$; and the hazard ratio was 0.51 (95\% CI, 0.27-0.96) in favor of CRS+SPIC, $\mathrm{P}=0.04$. An additional non-randomized comparative study between CRS+SPIC vs. systemic chemotherapy also demonstrated an improvement when using the combined approach (12). Median OS in this study was 32 months (95\% CI, 22-63) for CRS+SPIC treatment vs. 14 months (95\% CI, 6-25) for systemic chemotherapy, $\mathrm{P}=0.01$. The long-term results between systemic chemotherapy and CRS+SPIC also favor CRS+SPIC. The 5 -year survival in the non-randomized trial was $28 \%$ with CRS+SPIC but only $5 \%$ with systemic chemotherapy, albeit outdated 5-fluorouracil therapy (12). Yet in the more recent RCT, the difference was 33\% vs. $4 \%$. In the RCT, the systemic chemotherapy arm was oxaliplatin-based (10). Despite intensifying the systemic chemotherapy, the long-term survival was not increased. This underscores the fact that systemic chemotherapy has poor effect on isolated colorectal PM.

\section{Quality-of-life and cost-effectiveness}

In a follow-up study of the RCT, health-related quality of life (HRQOL) of the CRS+SPIC arm was better than a general reference population after 24 months (15). Numerically, the HRQOL for the systemic chemotherapy arm was lower. However, the sample sizes were too small to draw any firm conclusions about superiority of either arm. Nonetheless, one of the major criticisms of this combination treatment (CRS+SPIC) is that it is associated with increased morbidity, and an assumption is that it will impair the long-term HRQOL. This study was able to dismiss this concern as unfounded. The long-term survivors have a good HRQOL.
A quality adjusted life years (QALY) analysis was performed in the same study as the HRQOL (15). The QALY benefit in favor of CRS+SPIC with 3.8 QALYs was very near statistical significance $(\mathrm{P}=0.06)$; and the incremental cost-effectiveness ratio per QALY gained was between $£ 26,700-31,200$ ( $\$ 33,600-39,200)$ making the treatment cost-effective by most health-care systems.

\section{SPIC vs. HIPEC}

HIPEC has become a more widespread method for treating colorectal PM. There are two comparative studies performed between HIPEC and SPIC, but none are randomized. One case/control study demonstrated that HIPEC was superior to SPIC in both overall and diseasefree survival (5). The overall survival was 36.5 vs. 23.9 months, $\mathrm{P}=0.01$. Likewise, the disease-free survival was 22.8 vs. 13.0 months, $\mathrm{P}=0.02$. The grade 3-4 morbidity didn't differ statistically, but that may have been a power problem because numerically it was $37 \%$ vs. $19 \%$. The other study was a cohort study (16), where 69 HIPEC patients were compared to 57 SPIC patients that included all consecutive patients from the Uppsala center. The overall survival was 34 months with HIPEC and 25 months with SPIC, $\mathrm{P}=0.047$. However, in the multivariable analysis, it turned out that HIPEC was not superior to SPIC. Furthermore, the grade 3-4 morbidity did differ statistically, 30\% vs. $41 \%$ in favor of SPIC, $\mathrm{P}=0.02$.

\section{The future of SPIC}

SPIC treatment starts off in a similar manner as EPIC and then continues every 4-6 weeks for a total of 6 months of intraperitoneal chemotherapy treatment. EPIC has been widely used by itself, but also in conjunction with HIPEC. The use of SPIC for treating peritoneal metastases from colorectal cancer has been primarily a Swedish phenomenon. Consequently, research with this modality has been limited. Nonetheless, there is randomized data supporting its use; and furthermore, it is the only modality with a HRQOL and cost-effectiveness analysis within a randomized trial. With the setback of the Prodigy 7 trial (17), an interest in other modalities have been stimulated. While it is unlikely that SPIC will replace HIPEC, there may be a future for a combined approach. Anecdotally, one patient from the Uppsala center did receive combination HIPEC + SPIC with a very high peritoneal cancer index, and this patient is cured and has had no relapses for 16 years. The 
Table 1 Summary of sequential postoperative intraperitoneal chemotherapy (SPIC) outcomes

\begin{tabular}{ll}
\hline Endpoints & SPIC outcomes \\
\hline Grade 3-4 morbidity, \% & \\
Cohort 1 [11] & $41 \%$ \\
Phase 3/RCT setting [10] & $42 \%$ \\
Cohort 2 [16] & $30 \%$ \\
Case-control study [5] & $19 \%$ \\
Overall survival-median (after 5 years) & \\
Non-randomized comparison [12] & 32 months (28\%) \\
Phase 3/RCT setting [10] & 25 months (33\%) \\
Cohort 2 [16] & 25 months (18\%) \\
Case-control study [5] & 24 months (18\%) \\
$\begin{array}{l}\text { Disease-free survival-median } \\
\text { (after 5 years) }\end{array}$ & \\
Phase 3/RCT setting [10] & 12 months (17\%) \\
Cohort 2 [16] & 10 months (12\%) \\
Case-control study [5] & 13 months (N/A) \\
Health-related quality-of-life after & \\
24 months [15] & \\
Phase 3/RCT setting (EORTC mean & \\
Cost-effectiveness: phase 3/RCT [15] & \\
ICER/QALY increase (vs. systemic & Q \\
\hline
\end{tabular}

*, a Swedish healthy reference population scores 85 on their mean sum scores. SPIC, sequential postoperative intraperitoneal chemotherapy; RCT, randomized control trial, ICER, incremental cost-effectiveness ratio; QALY, quality-adjusted life year; chemo, chemotherapy, N/A, not available.

first step towards this combination is to investigate the combination of HIPEC + EPIC. Since SPIC starts off basically as an EPIC administration directly after CRS, this needs to be investigated in a randomized trial as a first step, which is something currently under consideration in the national HIPEC network in Sweden.

While the postoperative adjuvant treatment of SPIC barely survives in the shadow of HIPEC treatment, a new trial of repeated intraperitoneal chemotherapy in the neoadjuvant setting is being launched from Holland, the INTERACT trial (18). This may renew the repeat intraperitoneal chemotherapy modality as an option to pursue. The INTERACT trial is a phase 1 doseescalation study within the setting of non-resectable or high-volume PM of colorectal cancer. The most important advantage with the neoadjuvant setting compared to the adjuvant setting is that the abdomen has less adhesions and the problem of port dysfunction may be much less of an issue.

While SPIC has not had widespread use, there is evidence for its application (Table 1). Hopefully, the optimal perioperative and postoperative intraperitoneal chemotherapy treatment combination will be discerned in the coming years through more collaborative efforts. The goal is to improve the survival of patients with peritoneal metastases from colorectal cancer.

\section{Acknowledgments}

Funding: Bengt Ihre Fellowship funding and the Swedish Cancer Society project no. 150767 and 170206.

\section{Footnote}

Provenance and Peer Review: This article was commissioned by the Guest Editors (Paul H. Sugarbaker and Kurt Van der Speeten) for the focused issue "Intraperitoneal Chemotherapy for Peritoneal Metastases: HIPEC, EPIC, NIPEC, PIPAC and More" published in fournal of Gastrointestinal Oncology. This article has undergone external peer review.

Reporting Checklist: The authors have completed the Narrative Review Checklist. Available at http://dx.doi. org/10.21037/jgo-20-137

Conflicts of Interest: All authors have completed the ICMJE uniform disclosure form (available at http://dx.doi. org/10.21037/jgo-20-137). The focused issue was sponsored by the Peritoneal Surface Oncology Group International (PSOGI). The authors have no other conflicts of interest to declare.

Ethical Statement: The authors are accountable for all aspects of the work in ensuring that questions related to the accuracy or integrity of any part of the work are 
appropriately investigated and resolved.

Open Access Statement: This is an Open Access article distributed in accordance with the Creative Commons Attribution-NonCommercial-NoDerivs 4.0 International License (CC BY-NC-ND 4.0), which permits the noncommercial replication and distribution of the article with the strict proviso that no changes or edits are made and the original work is properly cited (including links to both the formal publication through the relevant DOI and the license). See: https://creativecommons.org/licenses/by-nc-nd/4.0/.

\section{References}

1. Dedrick RL, Flessner MF. Pharmacokinetic Problems in Peritoneal Drug Administration: Tissue Penetration and Surface Exposure. J Nat Cancer Inst 1997;89:480-7.

2. Auer RC, Sivajohanathan D, Biagi J, et al. Indications for hyperthermic intraperitoneal chemotherapy with cytoreductive surgery: a systematic review. Eur J Cancer 2020;127:76-95.

3. Lemoine L, Sugarbaker P, Van der Speeten K. Drugs, doses, and durations of intraperitoneal chemotherapy: standardising HIPEC and EPIC for colorectal, appendiceal, gastric, ovarian peritoneal surface malignancies and peritoneal mesothelioma. Int J Hyperthermia 2017;33:582-92.

4. Gockel I, Jansen-Winkeln B, Haase L, et al. Pressurized IntraPeritoneal Aerosol Chemotherapy (PIPAC) in patients with peritoneal metastasized colorectal, appendiceal and small bowel cancer. Tumori 2020;106:70-8.

5. Cashin PH, Graf W, Nygren P, et al. Intraoperative hyperthermic versus postoperative normothermic intraperitoneal chemotherapy for colonic peritoneal carcinomatosis: a case-control study. Ann Oncol 2012;23:647-52.

6. Cunliffe WJ, Sugarbaker PH. Gastrointestinal malignancy: Rationale for adjuvant therapy using early postoperative intraperitoneal chemotherapy. Br J Surg 1989;76:1082-90.

7. Graf W, Westlin JE, Påhlman L, et al. Adjuvant intraperitoneal 5-fluorouracil and intravenous leucovorin after colorectal cancer surgery: a randomized phase II placebo-controlled study. Int J Colorectal Dis 1994;9:35-9.

8. Weiber S, Graf W, Glimelius B, et al. Experimental colonic healing in relation to timing of 5 -fluorouracil therapy. Br J Surg 1994;81:1677-80.

9. Graf W, Ivarsson M, Gerdin B, et al. The influence of early postoperative intraperitoneal chemotherapy on human wound healing. J Surg Res 1994;57:394-400.

10. Cashin PH, Mahteme H, Spång N, et al. Cytoreductive surgery and intraperitoneal chemotherapy versus systemic chemotherapy for colorectal peritoneal metastases: A randomised trial. Eur J Cancer 2016;53:155-62.

11. Hansson J, Graf W, Påhlman L, et al. Postoperative adverse events and long-term survival after cytoreductive surgery and intraperitoneal chemotherapy. Eur J Surg Oncol 2009;35:202-8.

12. Mahteme H, Hansson J, Berglund A, et al. Improved survival in patients with peritoneal metastases from colorectal cancer: a preliminary study. Br J Cancer 2004;90:403-7.

13. Armstrong DK, Bundy B, Wenzel L, et al. Intraperitoneal cisplatin and paclitaxel in ovarian cancer. $\mathrm{N}$ Engl J Med 2006;354:34-43.

14. Hansson J. Loco-regional Treatment of Peritoneal Carcinomatosis: Survival, Morbidity and Quality of Life [PhD dissertation]. [Uppsala]: Acta Universitatis Upsaliensis; 2009. (Digital Comprehensive Summaries of Uppsala Dissertations from the Faculty of Medicine). Available online: http://urn.kb.se/resolve? urn=urn:nbn:se: uu:diva-107782

15. Cashin PH, Mahteme H, Syk I, et al. Quality of life and cost effectiveness in a randomized trial of patients with colorectal cancer and peritoneal metastases. Eur J Surg Oncol 2018;44:983-90.

16. Cashin PH, Graf W, Nygren P, et al. Cytoreductive surgery and intraperitoneal chemotherapy for colorectal peritoneal carcinomatosis: prognosis and treatment of recurrences in a cohort study. Eur J Surg Oncol 2012;38:509-15.

17. Quenet F, Elias D, Roca L, et al. A UNICANCER phase III trial of hyperthermic intra-peritoneal chemotherapy (HIPEC) for colorectal peritoneal carcinomatosis (PC): PRODIGE 7. J Clin Oncol 2018;36:LBA3503.

18. de Boer NL, Brandt-Kerkhof ARM, Madsen EVE, et al. Concomitant intraperitoneal and systemic chemotherapy for extensive peritoneal metastases of colorectal origin: protocol of the multicentre, open-label, phase I, doseescalation INTERACT trial. BMJ Open 2019;9:e034508.

Cite this article as: Cashin PH, Graf W. Sequential postoperative intraperitoneal chemotherapy for colorectal cancer with peritoneal metastases: a narrative review. J Gastrointest Oncol 2021;12(Suppl 1):S131-S135. doi: 10.21037/ jgo-20-137 